\title{
High-frequency magnon excitation due to femtosecond spin-transfer torques
}

\author{
Ulrike Ritzmann $\odot,{ }^{1,2,3,{ }^{*}}$ Pavel Baláž $\odot,{ }^{4,5}$ Pablo Maldonado $\odot,{ }^{1}$ Karel Carva $\odot,{ }^{4}$ and Peter M. Oppeneer $\oplus^{1,2}$ \\ ${ }^{1}$ Department of Physics and Astronomy, Uppsala University, Box 516, SE-75120 Uppsala, Sweden \\ ${ }^{2}$ Department of Physics, Freie Universität Berlin, Arnimallee 14, D-14195 Berlin, Germany \\ ${ }^{3}$ Dahlem Center of Complex Quantum Systems, Freie Universität Berlin, Arnimallee 14, D-14195 Berlin, Germany \\ ${ }^{4}$ Department of Condensed Matter Physics, Faculty of Mathematics and Physics, Charles University, Ke Karlovu 5 , \\ CZ 12116 Prague, Czech Republic \\ ${ }^{5}$ IT4 Innovations Center, VSB Technical University of Ostrava, 17 listopadu 15, CZ 70833 Ostrava-Poruba, Czech Republic
}

(Received 1 October 2019; revised manuscript received 3 April 2020; accepted 9 April 2020; published 18 May 2020)

\begin{abstract}
Femtosecond laser pulses can induce ultrafast demagnetization as well as generate bursts of hot-electron spin currents. In trilayer spin valves consisting of two metallic ferromagnetic layers separated by a nonmagnetic one, hot-electron spin currents excited by an ultrashort laser pulse propagate from the first ferromagnetic layer through the spacer, reaching the second magnetic layer. When the magnetizations of the two magnetic layers are noncollinear, this spin current exerts a torque on magnetic moments in the second ferromagnet. Since this torque is acting only within the subpicosecond timescale, it excites coherent high-frequency magnons, as recently demonstrated in experiments. Here, we calculate the temporal shape of the hot-electron spin currents using the superdiffusive transport model and simulate the response of the magnetic system to the resulting ultrashort spintransfer torque pulse by means of atomistic spin-dynamics simulations. Our results confirm that the acting spincurrent pulse is short enough to excite magnons with frequencies beyond $1 \mathrm{THz}$, a frequency range out of reach for current-induced spin-transfer torques. We demonstrate the formation of thickness-dependent standing spin waves during the first picoseconds after laser excitation. In addition, we vary the penetration depth of the spintransfer torque to reveal its influence on the excited magnons. Our simulations clearly show a suppression effect of magnons with short wavelengths already for penetration depths in the range of $1 \mathrm{~nm}$, confirming experimental findings reporting penetration depths below $2 \mathrm{~nm}$.
\end{abstract}

DOI: 10.1103/PhysRevB.101.174427

\section{INTRODUCTION}

The first experimental observation of ultrafast demagnetization due to femtosecond laser excitation in nickel was reported more than 20 years ago [1]. Since then, a variety of research activities have focused on studying the magnetization dynamics induced by intense, ultrashort laser pulses [2-4]. For the purpose of possible technological applications, especially, all-optical magnetization switching has become a topic of current research [5-9]. However, the relevant microscopic scattering processes and their interplay leading to ultrafast demagnetization are still under debate [10-17]. Laser-induced ultrafast demagnetization can also lead to spin-polarized currents of hot electrons [13,18-21]. Moreover, experiments have suggested that a single pulse of hot-electron spin currents without any assistance from laser heating induces ultrafast demagnetization of an adjacent magnetic layer [22-25]. Im-

\footnotetext{
*Corresponding author: ulrike.ritzmann@fu-berlin.de
}

Published by the American Physical Society under the terms of the Creative Commons Attribution 4.0 International license. Further distribution of this work must maintain attribution to the author(s) and the published article's title, journal citation, and DOI. Funded by Bibsam. portantly, it has been demonstrated [26,27] that femtosecond spin currents carried by hot electrons can exert spin-transfer torque (STT) [28-30] on a ferromagnet, leading to the rotation of magnetization or the excitation of high-frequency magnons [31] in a confined ferromagnetic structure.

Spin- and energy-resolved transport of laser-excited hot electrons can be described by the model of superdiffusive transport introduced by Battiato et al. [13,32]. The model is based on semiclassical equations of motion for electrons having nonthermal energies above the Fermi level and moving in two spin channels. A strong asymmetry of the electron velocities and lifetimes for the different spin channels in ferromagnetic metals leads to spin-polarized currents, following electron excitation, which can propagate across the nonmagnetic layer within femtoseconds and enter another magnetic layer. Alternatively, spin-dependent transport of hot electrons can also be described by a model based on the Boltzmann equation [33,34], which leads to similar results. If the magnetizations of both magnetic layers are noncollinear, the transverse part of the spin current (with respect to the local magnetization direction) will be absorbed by the ferromagnet and transformed into an STT, inducing magnetization dynamics [35-37]. These femtosecond STTs due to hot-electron spin currents can trigger the excitation of high-frequency magnons, which form standing spin waves in ultrathin magnetic layers $[31,38]$. 
Here, we present a theoretical study combining the superdiffusive spin-transport and atomistic spin-dynamics simulations [39-41] to describe the ultrafast magnon excitation in a metallic spin valve. We start by calculating the laserexcited spin current in the first ferromagnetic layer via the superdiffusive spin-transport theory [42] extended to describe the spin current propagating through the spacer and considering perpendicular alignment of the two ferromagnetic layers [37]. The excited spin current exerts STT on the second ferromagnetic layer, leading to magnetization dynamics within this layer. We describe this magnetization dynamics with atomistic spin-dynamics simulations. Particularly, we discuss the thickness-dependent magnon frequency spectra considering atomistic spin dynamics with exchange interactions beyond the nearest-neighbor approach determined by $a b$ initio methods. Furthermore, it has been shown experimentally that the components of the spin current transverse to the layer's magnetization are not absorbed directly at the interface between the nonmagnetic and magnetic layers; rather, they penetrate into the magnetic layer up to a distance of few nanometers $[31,43]$. Therefore, we consider the STT that not only acts on the first atomic layer of the magnetic material but affects also subsequent magnetic moments, taking into account the gradual decrease of STT. Consequently, we study the STT effect on the magnon spectra dependent on the considered penetration depth of the transverse spin current. Last, we analyze the time evolution of the frequency spectra. Our results reveal further insights in how to tailor the trilayer composition so that the desired magnonic contribution is enhanced.

This paper is organized as follows. In Sec. II we introduce the methods we have used. We outline both the basic approach to spin-torque calculation and atomistic spin-dynamics simulations. In Sec. III we present a description and discussion of our results. Finally, we conclude in Sec. IV.

\section{METHODS}

\section{A. Femtosecond spin-transfer torque terms due to laser excitation in FM1|NM|FM2 trilayers}

In our calculations, we assume a spin-valve-type magnetic trilayer of a structure $\mathrm{FM} 1\left(d_{1}\right)|\mathrm{NM}(d)| \mathrm{FM} 2\left(d_{2}\right)$, where FM1 and FM2 are metallic ferromagnetic layers and NM is a metallic nonmagnetic spacer. The setup is illustrated in Fig. 1. The numbers in the parentheses correspond to the widths of the layers. In our calculations we have used $d_{1}=16 \mathrm{~nm}$ and $d=4 \mathrm{~nm}$, and we have varied the thickness $d_{2}$. For both ferromagnetic layers we consider the same material, $\mathrm{Fe}$, while the nonmagnetic spacer is assumed to be made of $\mathrm{Cu}$. This trilayer system is excited by an ultrashort laser pulse acting on the left side of FM1. The demagnetization of FM1 leads to a time-delayed spin current arriving at FM2.

Two different models were proposed previously to explain the spin current reaching FM2. In one proposal the laser heats quickly the electrons in FM1, which transfer their energy to the spin system, causing magnon excitations. These magnons cause electronic spin accumulation in the nonmagnetic layer, which thermally diffuses through NM to reach FM2 [27,44,45]. The second proposal assumes that the laser pulse excites spin-polarized electrons in FM1. These hot,

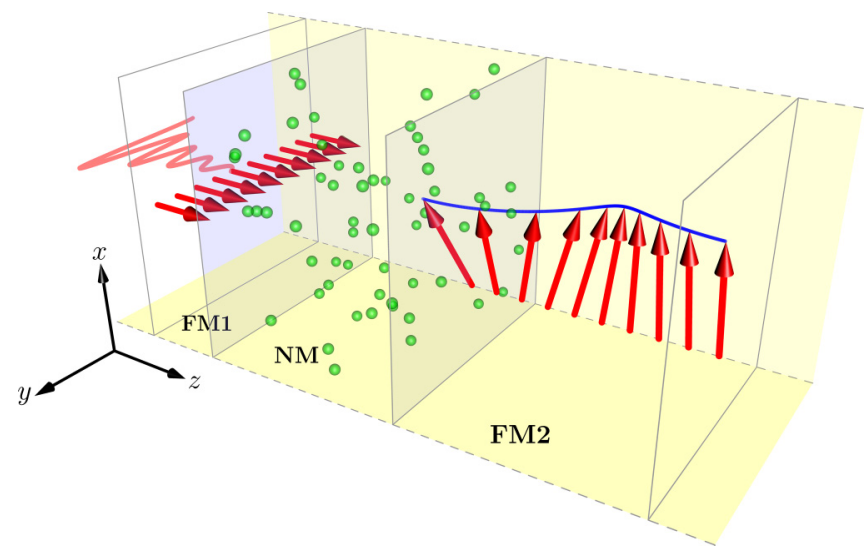

FIG. 1. Studied trilayer structure FM1|NM|FM2 made of two ferromagnetic layers (FM1 and FM2) separated by a nonmagnetic one (NM). Magnetic moments are presented by the red arrows. Layer FM1, which is assumed to have perpendicular magnetic anisotropy, is excited by a femtosecond laser pulse from the back of the device. A spin current of hot electrons (green balls) generated in FM1 is transmitted through the NM layer into FM2, where it exerts a spin-transfer torque on the magnetic moments, causing spin-wave excitations.

nonequilibrium electrons form a spin current that will quickly move through the NM spacer layer to reach FM2, as described by the superdiffusive transport model $[13,32]$. A major distinction between these two models lies in the timescale at which the spin angular momentum is transferred. The fastest superdifffusive electrons move with ballistic velocities from FM1 to FM2. This is consistent with experiments that detect an ultrafast injection and transfer of a spin current in metallic heterostructures $[19,24,46]$. For example, spin injection from a Ni layer into a Au layer was found to occur within about $40 \mathrm{fs}$ [46]. On the other hand, the timescale of the initial energy transfer process from hot electrons to hot magnons in FM1 has not been definitely established [3]. Hot magnons have been detected in laser-excited Co at $700 \mathrm{fs}$ [47], and they are likely present already at earlier times [48]. Thus, while the angular momentum transfer starting with hot magnons in FM1 and the subsequent diffusion through the NM layer are possible, this process is expected to occur on a timescale that is slower than superdiffusive spin currents.

In this work, we describe the resulting spin- and energyresolved transport of hot electrons from the first ferromagnetic layer through the normal metal using the superdiffusive transport model $[13,32]$ and describe the ensuing magnetization dynamics of the second ferromagnetic layer by means of atomistic spin-dynamics simulations (as outlined further below).

The main input parameters for the superdiffusive transport model are energy- and spin-dependent electron velocities and lifetimes, which are obtained from ab initio calculations $[49,50]$. We consider laser excitation of only the first ferromagnet. Consequently, the electrons from the $d$ band are excited into the $s p$ band above the Fermi level. In our calculations, we have assumed 12 uniformly distributed energy levels above the Fermi level with energy spacing $\Delta \epsilon=0.125 \mathrm{eV}$. Thus, electrons up to $1.5 \mathrm{eV}$ above the Fermi level are excited 


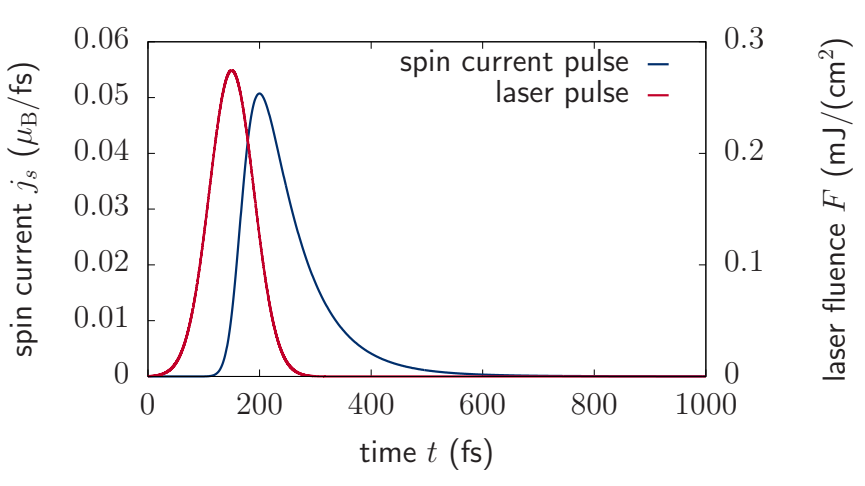

FIG. 2. Laser fluence of the laser pulse and calculated superdiffusive hot-electron spin current $j_{s}$ per atom entering the second FM as a function of time $t$. Note that the maximum of the laser pulse is at $t_{0}=150 \mathrm{fs}$.

by the laser pulse. We assume that the laser pulse populates the same electron density on each energy level. Moreover, the same number of electrons is populated in both spin channels.

The initial time dependence of the hot-electron distribution is given by the temporal shape of the laser pulse. We consider a Gaussian-shaped laser pulse, which dictates the time-dependent number of electrons on each energy level $\epsilon$ in spin channel $\sigma \in\{\uparrow, \downarrow\}$ being evolved as

$$
N_{\sigma}(t, z, \epsilon)=\bar{N}_{\sigma}(\epsilon, z) \frac{1}{\Delta \sqrt{2 \pi}} \exp \left\{\frac{\left(t-t_{0}\right)^{2}}{2 \Delta^{2}}\right\},
$$

where $\bar{N}_{\sigma}(\epsilon, z)$ is the average number of excited electrons at energy level $\epsilon$ with spin $\sigma$ at position $z$. Moreover, $t_{0}$ stands for the time-zero position of the pulse peak, while $\Delta$ is the pulse width. Here, we have assumed $\Delta=40 \mathrm{fs}$. In order to assume a finite penetration width of the laser $\ell$, we assume that the average number of excited electrons exponentially decreases with the distance from the left interface of the FM1 layer located at $z=0$,

$$
\bar{N}_{\sigma}(\epsilon, z)=\bar{N}_{0} \exp (-z / \ell),
$$

where $\bar{N}_{0}=\bar{N}(0, \epsilon)$ is the same for all energy levels, $\epsilon=\epsilon_{i}$. In our calculations we have used $\bar{N}_{0}=0.1$ electron per level, corresponding to a laser fluence $F=27.5 \mathrm{~mJ} \mathrm{~cm}^{-2}$ for $\mathrm{Fe}$. The laser penetration depth is assumed to be $\ell=15 \mathrm{~nm}$ in all layers. Moreover, the model assumes both gradual relaxation of high-energy electrons toward lower energy levels and generation of higher-order electrons due to elastic scattering of itinerant electrons on atoms.

Importantly, we consider the FM2 magnetization being perpendicular to the one of FM1. Furthermore, we suppose that the spin current generated and polarized by the FM1 layer is entirely absorbed by the FM2 layer and thus completely transformed into spin-transfer torque. This gives rise to a dampinglike spin-torque term exerted on magnetic moments. For more details on the spin-torque calculation methodology, see Ref. [37]. The resulting spin current entering the second ferromagnet has a duration of about $500 \mathrm{fs}$, and its calculated time-dependent profile is shown in Fig. 2.

\section{B. Atomistic spin-dynamics simulations}

For our spin-dynamics simulations we consider a thin iron layer with a bcc lattice. To describe the resulting dynamics we solve the Landau-Lifshitz-Gilbert (LLG) equation numerically [39],

$$
\begin{aligned}
\frac{\partial \mathbf{m}_{i}}{\partial t}= & -\frac{\gamma}{\mu_{\mathrm{s}}} \mathbf{m}_{i}(t) \times \mathbf{H}_{i}(t)+\alpha \mathbf{m}_{i}(t) \times \frac{\partial \mathbf{m}_{i}(t)}{\partial t} \\
& +\frac{\tau_{\mathrm{IF}}}{\mu_{\mathrm{s}}}(t, z) \mathbf{m}_{i}(t) \times \mathbf{m}_{i}(t) \times \hat{\mathbf{z}}
\end{aligned}
$$

for the (normalized) magnetic moments $\mathbf{m}_{i}$ of each atom in the iron layer at lattice position $i$. The equation consists of a precessional term of the normalized magnetic moment $\mathbf{m}_{i}=$ $\mathbf{M}_{i} / \mu_{\mathrm{s}}$ around its effective field $\mathbf{H}_{i}$ and a phenomenlogical relaxation term with damping constant $\alpha$ [51]. Moreover, we consider an additional dissipative term due to the femtosecond STT that acts like an antidamping torque term. $\gamma=1.76 \times$ $10^{11} \mathrm{~T} / \mathrm{s}$ denotes the gyromagnetic ratio, $\mu_{\mathrm{s}}=2.2 \mu_{\mathrm{B}}$ is the absolute value of the magnetic moment of each atom, and $\mu_{\mathrm{B}}=9.27 \times 10^{-24} \mathrm{~J} / \mathrm{T}$ is the Bohr magneton. The effective field $\mathbf{H}_{i}(t)$ is given by the derivative of the Hamiltonian with respect to the magnetic moment $\mathbf{m}_{i}$. We consider exchange interaction beyond the nearest-neighbor approach and an anisotropy including crystalline anisotropy, as well as a shape anisotropy given by the demagnetization field, and use the following Hamiltonian:

$$
\mathcal{H}=-\sum_{i<j} J_{i j} \mathbf{m}_{i} \cdot \mathbf{m}_{j}-\sum_{i}\left[k_{x}\left(m_{i}^{x}\right)^{2}+k_{z}\left(m_{i}^{z}\right)^{2}\right] .
$$

The exchange interaction between the magnetic moments at lattice nodes $i$ and $j$ in iron can alternate in sign depending on the distance of the two magnetic moments from each other. The values of the exchange interactions and details of their calculation can be found in Appendix A. The exchange interaction in metals like iron is long range. Consequently, a large number of neighboring shells are required to achieve converged spin-wave stiffnesses and magnon dispersions [52,53]. In our numerical simulations, we include exchange interactions up to the sixth neighboring shell, which corresponds to the distance of $2 a$, where $a$ is the lattice constant. This allows us to study the magnon dispersion in a realistic model for a bigger range of $q$ than we could using effective nearestneighbor interaction, and we can also include possible effects of the confinement of the system on the dispersion of the magnons. Note that exchange interactions at higher than sixth neighbors might still be relevant and affect the dispersion relation and the effective spin-wave stiffness. However, this will cause only a minor shift of the frequencies of the standing waves, but it will not have any further effect on the results. We present numerical simulations based on a comparable nearest-neighbor model with an effective nearest-neighbor exchange coupling in Appendix B. In addition to a different magnon dispersion, we observe a smaller-amplitude response of the excited magnons in the case of only nearest-neighbor interaction compared to the model with six neighboring shells. We mention furthermore that we study here the resulting magnetization dynamics due to the hot-electron spin currents without temperature effects. We expect only smaller quantitative changes of the dispersion and of the magnon lifetimes at 
room temperature, as confirmed experimentally, for example, by Qin et al. [54].

Due to the small thickness of the second ferromagnet, the demagnetization field due to the dipolar interaction of the magnetic moments causes a shape anisotropy which aligns the magnetic moment perpendicular to the $z$ direction and, therefore, perpendicular to the first magnetic layer. We consider a uniaxial anisotropy with a hard axis in the $z$ direction and an anisotropy constant of $k_{z}=-0.267 \mathrm{meV}$. Furthermore, we consider a magnetic anisotropy aligning the magnetic moments in the $x$ direction with an anisotropy constant of $k_{x}=0.00697 \mathrm{meV}$. Both values are taken from experiments by Razdolski et al. [31]. We initialize the magnetic moments parallel to the $x$ direction, which is taken as the magnetic ground state of the system.

The magnetic system is excited by femtosecond STT, which has been determined as described in the previous section. In the following, we use the same STT in all performed simulations, but we couple the STT in two different ways to the magnetic system. In our first calculations, we consider that the spin current $j_{\mathrm{s}}$ per atom is completely absorbed by the first magnetic plane,

$$
\tau_{\mathrm{IF}}(t, z)=j_{\mathrm{s}} \delta(z),
$$

where $z=0$ corresponds to the interface with the normal metal. In further calculations, we assume spin-current absorption within a characteristic penetration depth $\lambda_{\text {STT }}$ [55-57], and therefore, the STT acts on more than one atomic layer, and its spatial dependence is given by

$$
\tau_{\mathrm{IF}}(t, z)=\frac{j_{\mathrm{s}}}{\sum_{z} \exp \left(-\frac{z}{\lambda_{\mathrm{STT}}}\right)} \exp \left(-\frac{z}{\lambda_{\mathrm{STT}}}\right) .
$$

We perform atomistic spin-dynamics simulations by numerical integration of the LLG equation using the Heun method [39] with a time step of $0.1 \mathrm{fs}$. We study bcc Fe layers with a lattice constant $a=0.287 \mathrm{~nm}$ and different thicknesses $d_{2}$ ranging from 4.3 to $10 \mathrm{~nm}$. In addition, we consider a cross section of $4.3 \times 4.3 \mathrm{~nm}^{2}$ and apply periodic boundary conditions in the $x$ and $y$ directions. These boundary conditions are relevant to avoid effects in the $x$ and $y$ directions on the spin-wave dispersion relation.

\section{RESULTS}

To start with, we consider a femtosecond STT which is absorbed completely at the interface of the second ferromagnetic layer, and we use a thickness of $d_{2}=25 a=7.2 \mathrm{~nm}$ of the second ferromagnet. Using the atomistic spin-dynamics simulations we study the resulting magnetization dynamics during the first $100 \mathrm{ps}$. The ultrafast STT excites magnetization dynamics, leading to the creation of high-frequency magnons which propagate through the second magnetic layer and can be reflected multiple times before decaying.

In Fig. 3 we show the spatial profile of the magnetization components $\left\langle m_{y}(z)\right\rangle$ and $\left\langle m_{z}(z)\right\rangle$, which are transverse to the initial magnetization direction for different times. Here, $\langle\cdot\rangle$ denotes the average over the plane perpendicular to the propagation direction of the spin waves. At $500 \mathrm{fs}$ one can see that the signal is characterized by magnons with short wavelengths, and at 1 and $10 \mathrm{ps}$, one can clearly see that magnons

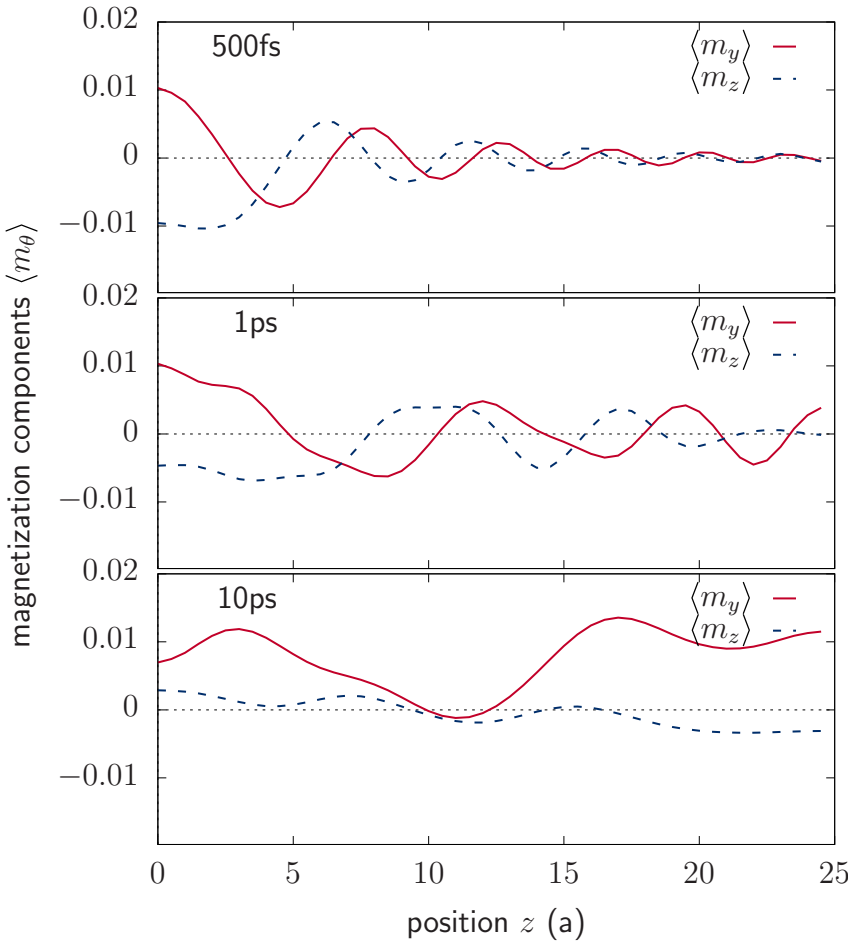

FIG. 3. Calculated spatial profile of the transverse magnetization components at different times.

with larger wavelengths become more relevant. This shows that a broad spectrum of frequencies is excited within the first picoseconds, but mainly, magnons with longer wavelengths remain at times beyond $10 \mathrm{ps}$.

\section{A. Formation of standing waves dependent on the thickness}

We find furthermore that standing waves are formed due to the small thickness of the ferromagnet. Only magnons with wavelengths fitting into the system dimensions remain after a few picoseconds (see below). For a further analysis, we study the time evolution of the averaged magnetization of the last layer $\left\langle\mathbf{m}\left(z=d_{2}\right)\right\rangle$ and perform a Fourier transformation in the time domain to determine the appearing frequencies. The magnetization components as a function of time are shown in the top panel of Fig. 4. The magnetization components oscillate very fast in the first picoseconds and then slower due to lower frequencies involved afterwards. The system remains excited over more than $100 \mathrm{ps}$. Note that the $y$ component of the magnetization oscillates stronger than the $z$ component. This is a consequence of the hard axis of the anisotropy in the $z$ direction, which suppresses larger amplitudes in that magnetization direction.

In the bottom panel of Fig. 4, the corresponding magnon amplitudes are shown as a function of frequency. To obtain the amplitudes we perform a discrete Fourier transformation of the last layer $\left(z=d_{2}\right)$ of $m^{+}(z, t)=\left\langle m_{y}(z, t)\right\rangle+i\left\langle m_{z}(z, t)\right\rangle$. We calculate the amplitude of the spin waves by considering the Fourier-transformed $m^{+}(\omega)$, which is given by

$$
m^{+}(\omega)=\frac{1}{N_{\text {steps }}} \sum_{n=1}^{N_{\text {steps }}} m^{+}\left(d_{2}, t_{n}\right) \exp \left(-i \omega t_{n}\right),
$$



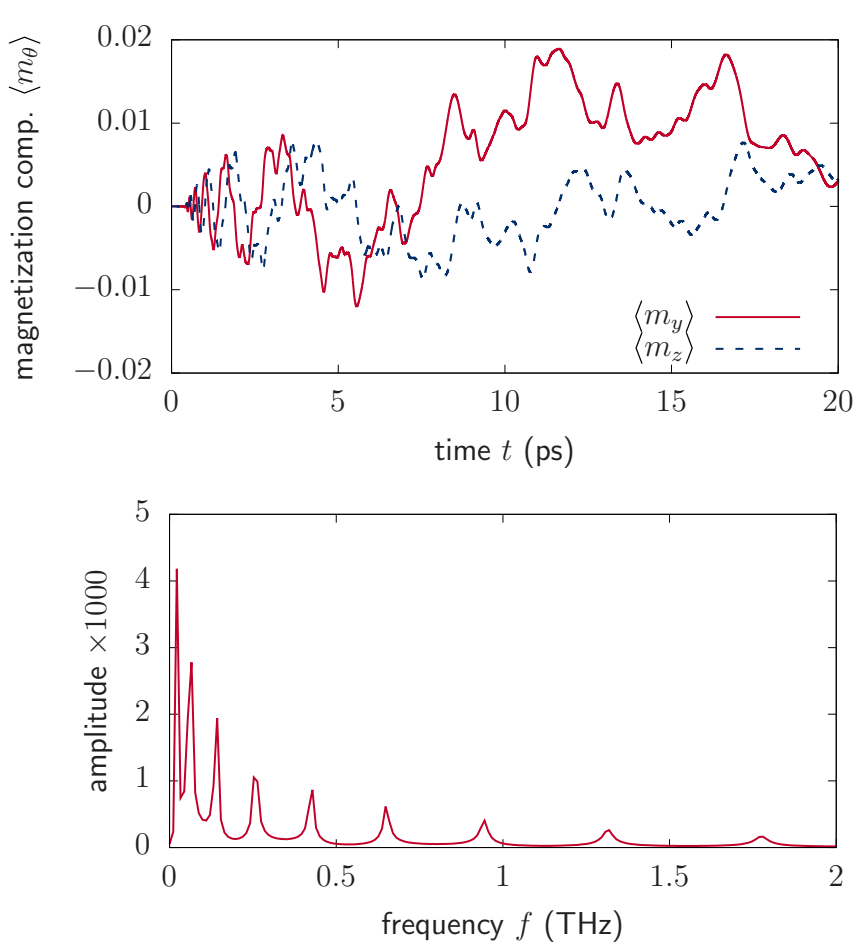

FIG. 4. Frequency spectra of the excited magnetization dynamics. Top: Calculated averaged magnetization components $\left\langle m_{y}\right\rangle$ and $\left\langle m_{z}\right\rangle$ of the last layer in the system. Bottom: Amplitude of the magnons as a function of frequencies obtained by Fourier transformation of the magnetization data.

where $N_{\text {steps }}$ is the number of time steps and $t_{n}=n \delta t$. The frequency spectrum reveals several peaks in the amplitude $\left|m^{+}(\omega)\right|$ corresponding to the frequencies of standing spin waves. For the Fourier transformation we integrate over a time interval of 100 ps with a step size of $1 \mathrm{fs}$. Note that especially after 10 ps lower frequencies dominate. Therefore, the amplitudes of low frequencies are larger than those of higher frequencies.

Standing spin waves are formed if the thickness of the system is a multiple of the wavelength and the wave vector $q_{z}$ is given by

$$
q_{z}=\frac{\pi n}{d_{2}}
$$

where $d_{2}$ is the thickness of the second ferromagnet as introduced before and $n=0,1,2, \ldots$ denotes the mode.

To obtain the corresponding frequencies, we determine the dispersion relation of the magnons analytically. For that purpose we consider the linearized LLG equation without damping and solve it analytically, as described in Ref. [58]. The solution of the resulting coupled set of equations is plane waves, and we obtain the Kittel formula [59]:

$$
h f=\hbar \omega=\sqrt{\left[2 k_{x}+J_{\text {eff }}(\mathbf{q})\right]\left[2 k_{x}-2 k_{z}+J_{\text {eff }}(\mathbf{q})\right]},
$$

where $J_{\text {eff }}(\mathbf{q})$ denotes the total contribution of the exchange interaction and is given by

$$
J_{\text {eff }}(\mathbf{q})=\sum_{k=1}^{6} J_{k}\left(N_{k}-\sum_{\theta_{k}} 2 \cos \left(\mathbf{q} \cdot \boldsymbol{\theta}_{k}\right)\right),
$$

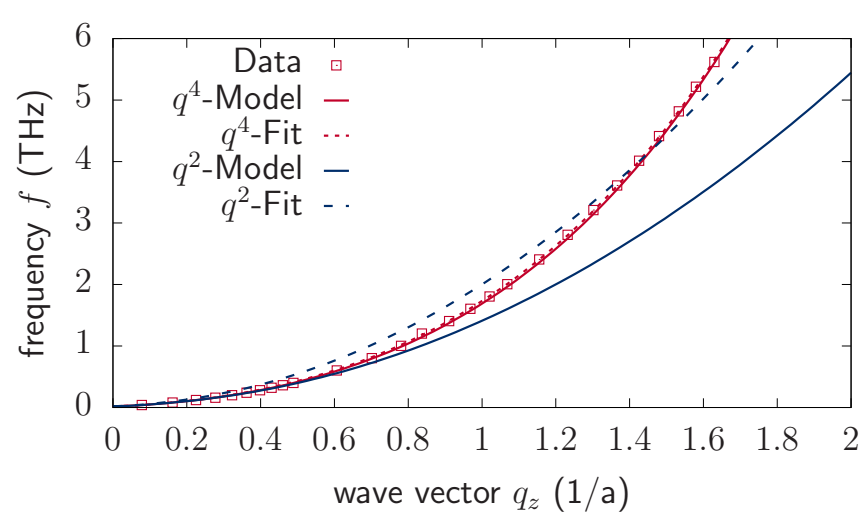

FIG. 5. Frequency of magnons as a function of the wave vector for one-dimensional spin-wave propagation. The red points show data obtained by our numerical simulations, whereas the red and blue lines show the analytical model based on Eqs. (9) and (11). The dotted red line shows a fit to the data points.

where $J_{k}$ is the exchange interaction with the $k$ th neighbor, $N_{k}$ is the number of $k$ th neighbors, and $\boldsymbol{\theta}_{k}$ are the distance vectors between the magnetic moment and the considered neighbor. We consider only spin-wave propagation in the $z$ direction with $\mathbf{q}=q_{z} \hat{\mathbf{z}}$ and simplify the expression by approximating $\cos x \approx 1-(1 / 2) x^{2}-(1 / 24) x^{4}$. We can then obtain a simplified expression for the exchange interaction

$$
J_{\text {eff }}=J_{\text {eff }, 1}\left(a q_{z}\right)^{2}+J_{\text {eff }, 2}\left(a q_{z}\right)^{4},
$$

with

$$
\begin{gathered}
J_{\mathrm{eff}, 1}=J_{1}+J_{2}+4 J_{3}+11 J_{4}+4 J_{5}+4 J_{6}, \\
J_{\mathrm{eff}, 2}=\frac{1}{48}\left(J_{1}+4 J_{2}+16 J_{3}+83 J_{4}+16 J_{5}+64 J_{6}\right) .
\end{gathered}
$$

Making this approximation, we obtain for the effective exchange constants $J_{\text {eff, } 1}=5.60 \mathrm{meV}$ and $J_{\text {eff }, 2}=1.42 \mathrm{meV}$. Note that especially, the value $J_{\text {eff, }, 1}$ is much smaller than the nearest-neighbor exchange interaction $(16.6 \mathrm{meV})$, which underlines the importance of properly taking the long-range exchange interactions into account. In Fig. 5 we show the analytical model in comparison to numerical data obtained by simulating monochromatic spin waves. The analytical result including a $q^{4}$ term of the exchange shows good agreement with the numerical data, whereas the approximation with only $q^{2}$ clearly deviates. A numerical fit of the data with a $q^{2}$ term gives a much higher effective exchange constant of $J_{\text {eff }, 1}^{\text {fit }-q^{2}}=7.90 \mathrm{meV}$. The fitting curve can describe overall the numerical results, but clear deviations occur. The obtained fitting parameter strongly deviates from the analytical model, demonstrating the importance of the higher-order corrections. A fit including the $q^{4}$ term shows small deviations at higher frequencies above $5 \mathrm{THz}$. In order to describe the dispersion relation with high precision, we also fit the dispersion relation and obtain small corrections for $J_{\text {eff, } 1}$ and $J_{\text {eff, } 2 \text {. The fitted }}$ values are $J_{\text {eff, }, 1}^{\mathrm{fit}}=5.72 \mathrm{meV}$ and $J_{\text {eff, } 2}^{\mathrm{fit}}=1.14 \mathrm{meV}$.

With this analytical model, we predict the spin-wave peak positions using the conditions for standing waves given by Eq. (8). In Fig. 6, we show the frequency spectra of the excited spin waves up to frequencies of $2 \mathrm{THz}$ for different 

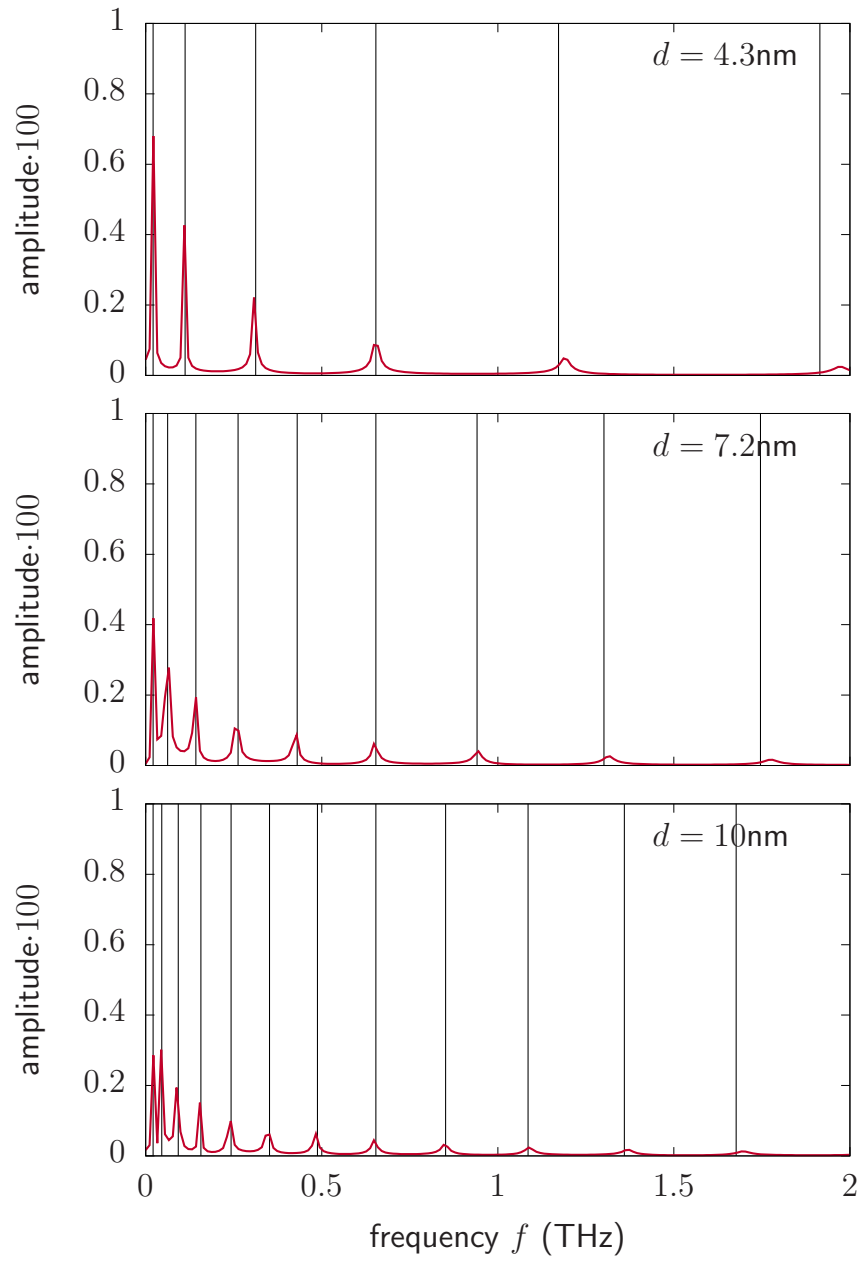

FIG. 6. Magnon frequency spectra for different thicknesses of FM2. The red lines show the spin-wave amplitude as a function of the frequency obtained by Fourier transformation in time of the magnetization of the last layer. The vertical black lines illustrate the predicted peak positions for standing waves with wave vectors given by Eq. (8).

thicknesses obtained by Fourier transformation as before. For a thickness of $d=4.3 \mathrm{~nm}$ one sees a strong peak at $q=0$ (peak with the lowest energy) corresponding to the ferromagnetic resonance (FMR) mode and four further peaks. The FMR peak appears in all shown cases at the same frequency, but by increasing the thickness of the film, the number of peaks within the $2-\mathrm{THz}$ range increases, as shown for thicknesses of 7.2 and $10 \mathrm{~nm}$. The predicted peak positions, which are illustrated as perpendicular lines, are in very good agreement with the numerically observed peaks. Note that the amplitudes of the single frequencies decrease for increasing thickness. The results demonstrate that in the time regime of $100 \mathrm{ps}$, only standing spin waves fulfilling Eq. (8) are relevant. Frequencies up to a few terahertz are excited through the femtosecond STT with a pulse duration of the excited hot-electron spin current of about $500 \mathrm{fs}$.

\section{B. Magnon distributions for different STT penetration depths}

To provide a more realistic description of the spin dynamics, we now consider a finite penetration depth of the trans-

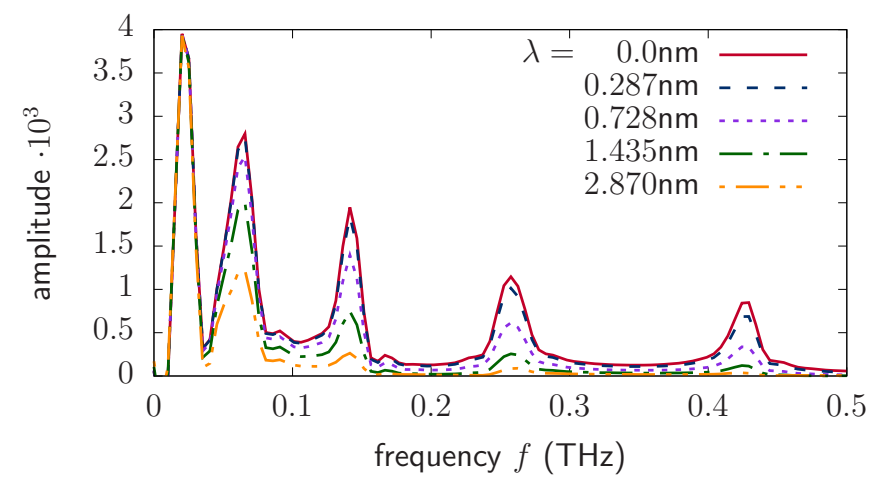

FIG. 7. Calculated magnon amplitudes as a function of the frequency for different penetration depths $\lambda_{\mathrm{s}}$.

verse spin. Large penetration depths suppress high-frequency magnons, and therefore, recent experiments investigated the maximum frequencies that can be excited to determine the upper bound of the penetration depth. Here, we will explore the modifications of the frequency-dependent amplitudes of the excited magnons due to a finite penetration depth. We perform simulations assuming an STT that enters the film with a characteristic penetration depth as described by Eq. (6). As for the case of full absorption at the interface, we use a film thickness of $d_{2}=25 a=7.2 \mathrm{~nm}$ for the second ferromagnet and perform our numerical simulation for a time interval of $100 \mathrm{ps}$ for the average magnetization of the last layer. We study the influence of the penetration depth in a range of $\lambda_{\mathrm{s}}$ equal to zero up to almost $3.0 \mathrm{~nm}$.

The resulting spin-wave amplitudes as a function of the frequency for different penetration depths are shown in Fig. 7. For comparison, we also include the obtained data from zeropenetration depth, $\lambda_{\mathrm{s}}=0$. The positions of the peaks are the same in all cases, as shown in Fig. 7, but the amplitudes for each peak strongly differ. The amplitude of the FMR mode, corresponding to $q_{z}=0$, is almost the same for the different penetration depths. On the other hand, already at the first spin-wave mode, the amplitude decreases significantly for the highest penetration depth. The amplitude is reduced by more than a factor of 2 for the largest value of $\lambda_{s}$, and here, the penetration corresponds to $1 / 5$ of the wavelength of the mode. For the largest penetration depth modes with $n \geqslant 4$ are no longer excited. The penetration depth in that case is larger than half of the wavelength of the magnon modes. For smaller values of the penetration depth, higher modes are still excited, but the amplitudes of the modes are strongly suppressed. A large penetration depth leads to an almost complete suppression of magnons with a wavelength $\lambda \leqslant 2 \lambda_{\text {STT }}$, but it can also cause a reduction of the amplitudes of the magnons with a wavelength larger than $2 \lambda_{\text {STT }}$. Although these results are in general agreement with experimental observations, they show that the determination of an upper limit for $\lambda_{\mathrm{STT}}$ is rather difficult due to a strong suppression for all modes with finite wavelength.

\section{Time evolution of the frequency spectra}

As the next step, we investigate the temporal evolution of the excited magnon spectra. Instead of calculating a Fourier 


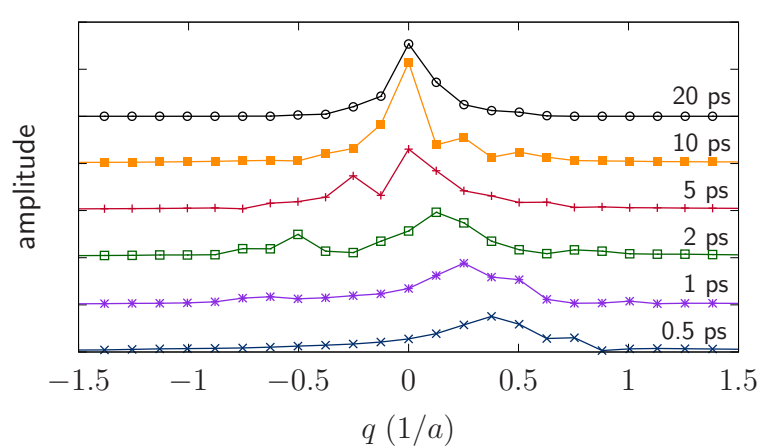

FIG. 8. Calculated spin-wave amplitudes as a function of wave vector $q_{z}$ obtained by space-domain Fourier transformation at different times. For better visualization of the occurring peaks in the Fourier spectrum, the data at different times are shifted upwards with increasing time.

transformation in the time domain, we perform a Fourier transformation now in the space domain and obtain the amplitudes as a function of the wave vector of the modes. To obtain the amplitudes we perform a discrete Fourier transformation of $m^{+}(z, t)$ at different times $t$. We calculate the amplitude of the spin waves by considering $\left|m^{+}\left(q_{z}, t\right)\right|$, which is given by the Fourier transformation

$$
m^{+}\left(q_{z}, t\right)=\frac{1}{N_{\mathrm{L}}} \sum_{z}^{N_{\mathrm{L}}} m^{+}(z, t) \exp \left(i q_{z} z\right)
$$

where the summation runs over all layers in FM2. The results are shown in Fig. 8. At $500 \mathrm{fs}$, a broad spectrum of frequencies with mainly positive wave vectors propagates through the system. At this time the spin waves have not reached the end of the system, and therefore, no reflection has occurred. The data show that the maximum in the amplitudes occurs around $q \approx 0.4(1 / a)$, which corresponds to a frequency of about $1 \mathrm{THz}$. But also higher modes are excited with wave vectors of $q \approx 0.8(1 / a)$ and a frequency of about $4.5 \mathrm{THz}$. At $1 \mathrm{ps}$, the maximum amplitude is already shifted to lower wave vectors, and the highest frequencies are reflected, and contributions with negative wave vectors are forming. At later times, beyond $5 \mathrm{ps}$, one can clearly see that modes with lower wave vectors start to dominate. Spin waves with larger wave vectors and, consequently, higher frequencies decay strongly within the first 10 ps. At 20 ps the distribution is almost symmetric due to a dephasing of the coherent magnon excitation.

As the last step, we want to compare our observation to the lifetimes of the frequencies due to Gilbert damping. To describe the frequency-dependent lifetimes due to Gilbert damping in the magnetic layer, we consider again the linearized LLG equations, but now we include also the damping contributions. We solve the coupled set of equations considering plane waves as a solution of the system. The imaginary part of the eigenvalue corresponds to the frequency of the system, and the real value is linked to the frequency-dependent lifetime of the spin waves. Note that the damping term also modifies the frequency of the magnons, which, however, becomes relevant only for larger damping values. The corrected frequency $\omega^{\prime}$ is

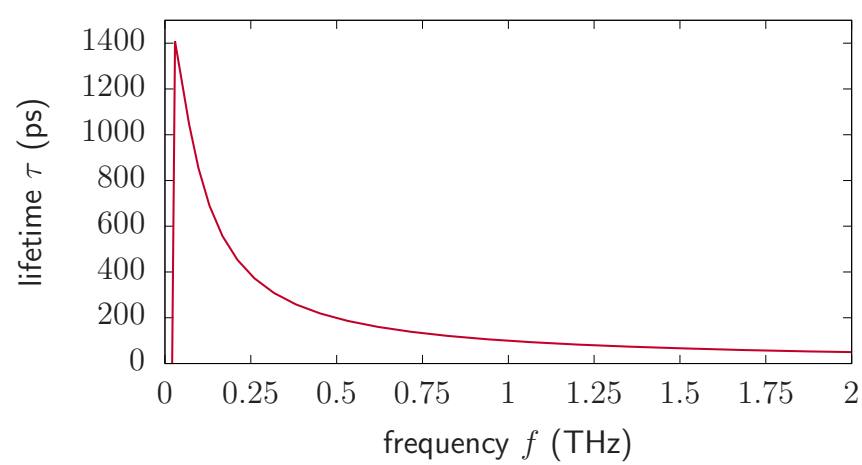

FIG. 9. Lifetime of magnons as a function of the frequency given by Eq. (16) and a damping constant of $\alpha=0.01$.

then given by

$$
\hbar \omega^{\prime}=\sqrt{(\hbar \omega)^{2}-\left(\frac{\alpha}{1+\alpha^{2}}\right)^{2}\left(J_{\text {eff }}(\mathbf{q})+2 k_{x}-k_{z}\right)} .
$$

The lifetime $\tau$ of the spin waves due to Gilbert damping describes an exponential decay of the modes after excitation and is strongly frequency dependent. We obtain for the wavevector-dependent lifetime

$$
\tau(\mathbf{q})=\frac{1}{\frac{\alpha}{1+\alpha^{2}}\left[J_{\mathrm{eff}}(\mathbf{q})+2 k_{x}+k_{z}\right]} .
$$

The resulting lifetime as a function of the frequency is shown in Fig. 9. Since we use a very small damping value of $\alpha=$ 0.01 , we neglect the frequency correction and use $\left(1+\alpha^{2}\right)$ $\approx 1$. The lifetime for larger frequencies is significantly reduced compared to the lifetime of magnons with low frequencies. Magnons with frequencies above $1 \mathrm{THz}$ decay within $100 \mathrm{ps}$, whereas magnons with lower frequencies can have lifetimes above $1 \mathrm{~ns}$.

The long lifetimes for the spin waves with low frequencies are in good agreement with our numerical observations and also with experimental findings. But although spin waves with higher frequencies have a much lower lifetime than the ones with lower frequencies, our numerical results indicate an even faster decay of these high-frequency modes. This could possibly be explained by nonlinear processes, which would even be enhanced at elevated temperatures.

\section{CONCLUSION}

To summarize, we have studied theoretically the laserinduced excitation of terahertz $(\mathrm{THz})$ magnons in $\mathrm{Fe}|\mathrm{Cu}| \mathrm{Fe}$ trilayer structures with two ferromagnetic materials with perpendicular magnetization orientations. We used a theoretical model to describe superdiffusive hot-electron transport leading to ultrafast spin-current transfer. These spin-current bursts excite $\mathrm{THz}$ magnons in the adjacent second ferromagnet via an ultrafast spin-transfer torque. We combine our model with atomistic spin-dynamics simulations including exchange interactions beyond nearest-neighbor interactions to describe the magnetization dynamics in the second ferromagnet on the atomic scale. In this way, we demonstrate the excitation of $\mathrm{THz}$ magnons and the formation of standing spin waves within the first picoseconds, as well as the larger lifetimes of 
the lowest-frequency modes, in good agreement with experimental observations. We analyze how the magnonic distribution depends on the penetration depth of the spin-transfer torque, and we find a complete suppression of magnons with wavelength $\lambda \leqslant 2 \lambda_{\text {STT }}$. The decrease in magnon mode population with frequency (studied within the first $100 \mathrm{ps}$ ) is apparently significantly faster for longer penetration depths. We confirm that STT penetration depth has to be smaller than $1 \mathrm{~nm}$ in order to achieve a significant occupation of the fourth magnon mode, as observed experimentally [31].

Our results demonstrate that laser-induced hot-electron spin currents offer a new pathway to excite high-frequency magnons in the $\mathrm{THz}$ regime, which allows for new design concepts for ultrafast spintronics and high-frequency magnonics applications. The developed theory can be used to tailor the trilayer composition so that the desired magnonic contribution is enhanced.

\section{ACKNOWLEDGMENTS}

We thank J. Hurst and A. Melnikov for valuable discussions. This work was supported by the Deutsche Forschungsgemeinschaft via Grants No. RI 2891/1-1, No. RI 2891/21, and via TRR 227 "Ultrafast Spin Dynamics"; by the Swedish Research Council (SE); and by the European Regional Development Fund in the IT4Innovations national supercomputing center-path to exascale project (Project No. CZ.02.1.01/0.0/0.0/16_013/0001791) within the Operational Programme Research, Development and Education; and by the Czech Science Foundation (Grant No. 18-07172S). We further acknowledge support from the Knut and Alice Wallenberg Foundation (Grant No. 2015.0060) and the Swedish National Infrastructure for Computing (SNIC). This work was supported by the Ministry of Education, Youth and Sports through the Large Infrastructures for Research, Experimental Development and Innovations project "IT4Innovations National Supercomputing Center - LM2015070.”

\section{APPENDIX A: EXCHANGE INTERACTIONS}

Exchange interaction constants can be calculated using infinitesimal spin rotations [60] or frozen spin-wave total energy calculations [53]. Here, we use the expression of Liechtenstein et al. [60], which reads

$$
\begin{aligned}
J_{i j}= & \frac{1}{\pi} \operatorname{Im} \int_{-\infty}^{E_{F}} d E \int_{\Omega_{i}} d \boldsymbol{r} \int_{\Omega_{j}} d \boldsymbol{r}^{\prime} \\
& \times B_{\mathrm{xc}}(\boldsymbol{r}) G^{\uparrow}\left(\boldsymbol{r}, \boldsymbol{r}^{\prime}, E^{+}\right) B_{\mathrm{xc}}\left(\boldsymbol{r}^{\prime}\right) G^{\downarrow}\left(\boldsymbol{r}^{\prime}, \boldsymbol{r}, E^{-}\right),
\end{aligned}
$$

where $G^{\sigma}$ is the spin-dependent Green's function with spin $\sigma \in\{\uparrow, \downarrow\}, B_{\mathrm{xc}}$ is the magnetic field from the exchangecorrelation potential, $\Omega_{i}$ is the volume of a sphere with the center in the $i$ th atom position, and $E^{ \pm}=\lim _{\alpha \rightarrow 0} E \pm i \alpha$, with $i=\sqrt{-1}$. Figure 10 shows the obtained exchange interactions for a bcc Fe lattice.

In our atomistic spin-dynamics simulations, we include exchange interaction up to the sixth neighboring shell, which corresponds to a distance of the neighbors of up to $2 a$.

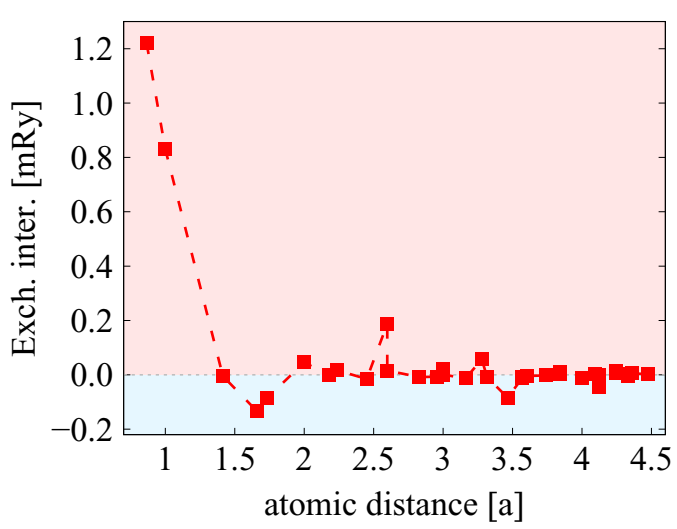

FIG. 10. Exchange interactions for the six nearest neighbors calculated for a bcc Fe lattice as a function of the distance between the atoms. The distance is given in units of the Fe lattice constant $a$.

\section{APPENDIX B: COMPARISON TO THE NEAREST-NEIGHBOR APPROXIMATION}

In the following, we will compare our results presented in Sec. III A with a simplified model considering only an effective nearest-neighbor approximation with an exchange coupling of $J_{\text {eff, } 1}=5.60 \mathrm{meV}$ and the same anisotropy constants. We study a bcc lattice with a thickness of $d_{2}=7.2 \mathrm{~nm}$ and apply a femtosecond STT acting on the first layer of FM2.

The resulting spin-wave spectrum obtained by a discrete Fourier transformation in the time domain over $100 \mathrm{ps}$ is shown in Fig. 11. The FMR peak occurs at the same frequency as before, but the peak positions of the other standing waves is shifted to lower frequencies compared to the results obtained with the long-range interaction model [see Fig. 4(b)]. The differences in the frequencies of the two models for the standing waves increase with increasing frequency due to the differences in the magnon dispersion relations of the two models. The black lines show the predicted peak positions for the standing waves in the nearest-neighbor model, and

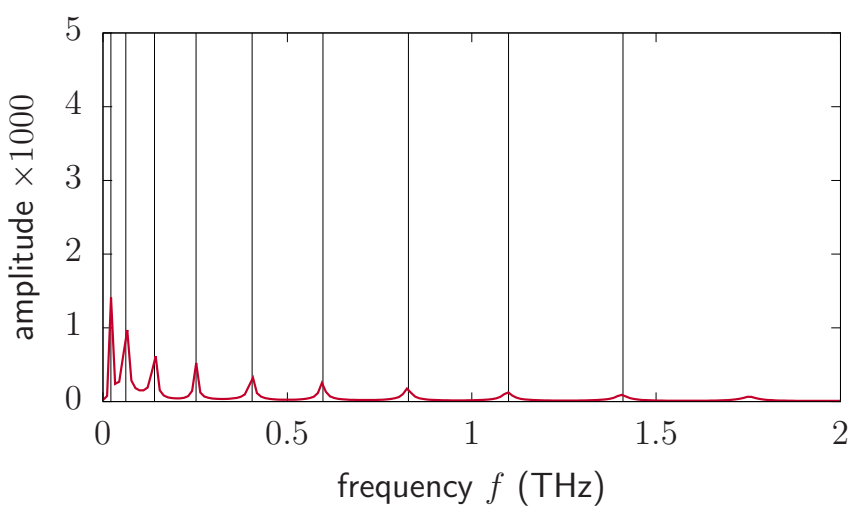

FIG. 11. Magnon frequency spectra computed for the nearestneighbor approximation to the exchange interactions. The red line shows the spin-wave amplitude as a function of the frequency obtained by a time-domain Fourier transformation of the magnetization of the last layer. The vertical black lines illustrate the predicted peak positions for standing waves with wave vectors given by Eq. (8). 
the obtained results show good agreement with the analytical predictions, when assuming only nonzero $J_{\text {eff, } 1}$.

Furthermore, the amplitudes of the standing spin waves strongly deviate between the two models. We obtain smaller amplitudes in the nearest-neighbor model, indicating a weaker response of the magnetic system to the ultrafast spin-current excitation. This is plausible since the exchange interaction with more distant neighbors allows for a faster penetration of the excitation into FM2 and, consequently, the spin-wave amplitudes can be increased.
[1] E. Beaurepaire, J.-C. Merle, A. Daunois, and J.-Y. Bigot, Phys. Rev. Lett. 76, 4250 (1996).

[2] J. Walowski and M. Münzenberg, J. Appl. Phys. 120, 140901 (2016)

[3] K. Carva, P. Baláž, and I. Radu, in Handbook of Magnetic Materials (Elsevier, Amsterdam, 2017), Vol. 26, pp. 291-463.

[4] G. Malinowski, N. Bergeard, M. Hehn, and S. Mangin, Eur. Phys. J. B 91, 98 (2018).

[5] C. D. Stanciu, F. Hansteen, A. V. Kimel, A. Kirilyuk, A. Tsukamoto, A. Itoh, and T. Rasing, Phys. Rev. Lett. 99, 047601 (2007).

[6] I. Radu, K. Vahaplar, C. Stamm, T. Kachel, N. Pontius, H. Dürr, T. A. Ostler, J. Barker, R. F. L. Evans, R. W. Chantrell, A. Tsukamoto, A. Itoh, A. Kirilyuk, T. Rasing, and A. V. Kimel, Nature (London) 472, 205 (2011).

[7] C.-H. Lambert, S. Mangin, B. S. D. C. S. Varaprasad, Y. K. Takahashi, M. Hehn, M. Cinchetti, G. Malinowski, K. Hono, Y. Fainman, M. Aeschlimann, and E. E. Fullerton, Science 345, 1337 (2014).

[8] M. L. M. Lalieu, M. J. G. Peeters, S. R. R. Haenen, R. Lavrijsen, and B. Koopmans, Phys. Rev. B 96, 220411(R) (2017).

[9] R. B. Wilson, Y. Yang, J. Gorchon, C.-H. Lambert, S. Salahuddin, and J. Bokor, Phys. Rev. B 96, 045105 (2017).

[10] B. Koopmans, J. J. M. Ruigrok, F. Dalla Longa, and W. J. M. de Jonge, Phys. Rev. Lett. 95, 267207 (2005).

[11] E. Carpene, E. Mancini, C. Dallera, M. Brenna, E. Puppin, and S. De Silvestri, Phys. Rev. B 78, 174422 (2008).

[12] B. Koopmans, G. Malinowski, F. Dalla Longa, D. Steiauf, M. Fähnle, T. Roth, M. Cinchetti, and M. Aeschlimann, Nat. Mater. 9, 259 (2010).

[13] M. Battiato, K. Carva, and P. M. Oppeneer, Phys. Rev. Lett. 105, 027203 (2010).

[14] M. Fähnle and C. Illg, J. Phys.: Condens. Matter 23, 493201 (2011).

[15] K. Carva, M. Battiato, and P. M. Oppeneer, Phys. Rev. Lett. 107, 207201 (2011).

[16] K. Carva, M. Battiato, D. Legut, and P. M. Oppeneer, Phys. Rev. B 87, 184425 (2013).

[17] K. Krieger, J. K. Dewhurst, P. Elliott, S. Sharma, and E. K. U. Gross, J. Chem. Theory Comput. 11, 4870 (2015).

[18] G. Malinowski, F. Dalla Longa, J. H. H. Rietjens, P. V. Paluskar, R. Huijink, H. J. M. Swagten, and B. Koopmans, Nat. Phys. 4, 855 (2008).

[19] A. Melnikov, I. Razdolski, T. O. Wehling, E. T. Papaioannou, V. Roddatis, P. Fumagalli, O. Aktsipetrov, A. I. Lichtenstein, and U. Bovensiepen, Phys. Rev. Lett. 107, 076601 (2011).

[20] D. Rudolf, C. La-O-Vorakiat, M. Battiato, R. Adam, J. M. Shaw, E. Turgut, P. Maldonado, S. Mathias, P. Grychtol, H. T. Nembach, T. J. Silva, M. Aeschlimann, H. C. Kapteyn, M. M. Murnane, C. M. Schneider, and P. M. Oppeneer, Nat. Commun. 3, 1037 (2012).
[21] A. Alekhin, I. Razdolski, N. Ilin, J. P. Meyburg, D. Diesing, V. Roddatis, I. Rungger, M. Stamenova, S. Sanvito, U. Bovensiepen, and A. Melnikov, Phys. Rev. Lett. 119, 017202 (2017).

[22] A. Eschenlohr, M. Battiato, P. Maldonado, N. Pontius, T. Kachel, K. Holldack, R. Mitzner, A. Föhlisch, P. M. Oppeneer, and C. Stamm, Nat. Mater. 12, 332 (2013).

[23] B. Vodungbo, B. Tudu, J. Perron, R. Delaunay, L. Müller, M. H. Berntsen, G. Grübel, G. Malinowski, C. Weier, J. Gautier, G. Lambert, P. Zeitoun, C. Gutt, E. Jal, A. H. Reid, P. W. Granitzka, N. Jaouen, G. L. Dakovski, S. Moeller, M. P. Minitti, A. Mitra, S. Carron, B. Pfau, C. von Korff Schmising, M. Schneider, S. Eisebitt, and J. Lüning, Sci. Rep 6, 18970 (2016).

[24] N. Bergeard, M. Hehn, S. Mangin, G. Lengaigne, F. Montaigne, M. L. M. Lalieu, B. Koopmans, and G. Malinowski, Phys. Rev. Lett. 117, 147203 (2016).

[25] Y. Xu, M. Deb, G. Malinowski, M. Hehn, W. Zhao, and S. Mangin, Adv. Mater 29, 1703474 (2017).

[26] A. J. Schellekens, K. C. Kuiper, R. R. J. C. de Wit, and B. Koopmans, Nat. Commun. 5, 4333 (2014).

[27] G.-M. Choi, B.-C. Min, K.-J. Lee, and D. G. Cahill, Nat. Commun. 5, 4334 (2014).

[28] J. C. Slonczewski, J. Magn. Magn. Mater 159, L1 (1996).

[29] L. Berger, Phys. Rev. B 54, 9353 (1996).

[30] J. C. Slonczewski, J. Magn. Magn. Mater 247, 324 (2002).

[31] I. Razdolski, A. Alekhin, N. Ilin, J. P. Meyburg, V. Roddatis, D. Diesing, U. Bovensiepen, and A. Melnikov, Nat. Commun. 8, 15007 (2017).

[32] M. Battiato, K. Carva, and P. M. Oppeneer, Phys. Rev. B 86, 024404 (2012).

[33] D. M. Nenno, S. Kaltenborn, and H. C. Schneider, Phys. Rev. B 94, 115102 (2016).

[34] D. M. Nenno, B. Rethfeld, and H. C. Schneider, Phys. Rev. B 98, 224416 (2018).

[35] M. D. Stiles and A. Zangwill, Phys. Rev. B 66, 014407 (2002).

[36] J. Barnaś, A. Fert, M. Gmitra, I. Weymann, and V. K. Dugaev, Phys. Rev. B 72, 024426 (2005).

[37] P. Baláž, M. Žonda, K. Carva, P. Maldonado, and P. M. Oppeneer, J. Phys.: Condens. Matter 30, 115801 (2018).

[38] H. Ulrichs and I. Razdolski, Phys. Rev. B 98, 054429 (2018).

[39] U. Nowak, in Micromagnetism, edited by H. Kronmüller, Handbook of Magnetism and Advanced Magnetic Materials Vol. 2 (Wiley, Chichester, 2007), pp. 858-876.

[40] B. Skubic, J. Hellsvik, L. Nordstrom, and O. Eriksson, J. Phys.: Condens. Matter 20, 315203 (2008).

[41] R. F. L. Evans, W. J. Fan, P. Chureemart, T. A. Ostler, M. O. A. Ellis, and R. W. Chantrell, J. Phys.: Condens. Matter 26, 103202 (2014).

[42] M. Battiato, P. Maldonado, and P. M. Oppeneer, J. Appl. Phys. 115, 172611 (2014). 
[43] M. L. M. Lalieu, P. L. J. Helgers, and B. Koopmans, Phys. Rev. B 96, 014417 (2017).

[44] G.-M. Choi and D. G. Cahill, Phys. Rev. B 90, 214432 (2014).

[45] E. G. Tveten, A. Brataas, and Y. Tserkovnyak, Phys. Rev. B 92, 180412(R) (2015).

[46] M. Hofherr, P. Maldonado, O. Schmitt, M. Berritta, U. Bierbrauer, S. Sadashivaiah, A. J. Schellekens, B. Koopmans, D. Steil, M. Cinchetti, B. Stadtmüller, P. M. Oppeneer, S. Mathias, and M. Aeschlimann, Phys. Rev. B 96, 100403(R) (2017).

[47] E. Turgut, D. Zusin, D. Legut, K. Carva, R. Knut, J. M. Shaw, C. Chen, Z. Tao, H. T. Nembach, T. J. Silva, S. Mathias, M. Aeschlimann, P. M. Oppeneer, H. C. Kapteyn, M. M. Murnane, and P. Grychtol, Phys. Rev. B 94, 220408(R) (2016).

[48] S. Eich, M. Plötzing, M. Rollinger, S. Emmerich, R. Adam, C. Chen, H. C. Kapteyn, M. M. Murnane, L. Plucinski, D. Steil, B. Stadtmüller, M. Cinchetti, M. Aeschlimann, C. M. Schneider, and S. Mathias, Sci. Adv. 3, e1602094 (2017).

[49] V. P. Zhukov, E. V. Chulkov, and P. M. Echenique, Phys. Rev. B 72, 155109 (2005).
[50] V. P. Zhukov, E. V. Chulkov, and P. M. Echenique, Phys. Rev. B 73, 125105 (2006).

[51] T. Gilbert, IEEE Trans. Magn 40, 3443 (2004).

[52] M. Pajda, J. Kudrnovský, I. Turek, V. Drchal, and P. Bruno, Phys. Rev. B 64, 174402 (2001).

[53] S. V. Halilov, H. Eschrig, A. Y. Perlov, and P. M. Oppeneer, Phys. Rev. B 58, 293 (1998).

[54] H. J. Qin, K. Zakeri, A. Ernst, and J. Kirschner, Phys. Rev. Lett. 118, 127203 (2017).

[55] M. Zwierzycki, Y. Tserkovnyak, P. J. Kelly, A. Brataas, and G. E. W. Bauer, Phys. Rev. B 71, 064420 (2005).

[56] P. Baláž, M. Zwierzycki, and J. Barnaś, Phys. Rev. B 88, 094422 (2013).

[57] K.-W. Kim, Phys. Rev. B 99, 224415 (2019).

[58] U. Ritzmann, D. Hinzke, and U. Nowak, Phys. Rev. B 89, 024409 (2014).

[59] C. Herring and C. Kittel, Phys. Rev. 81, 869 (1951).

[60] A. I. Liechtenstein, M. I. Katsnelson, V. P. Antropov, and V. A. Gubanov, J. Magn. Magn. Mater. 67, 65 (1987). 